\title{
On the Birkhoff Quadrature Formulas Using Even and Odd Order of Derivatives
}

\author{
S. Hatami, ${ }^{1}$ S. M. Hashemiparast, ${ }^{1}$ and S. Shateyi ${ }^{2}$ \\ ${ }^{1}$ Department of Applied Mathematics, K. N. Toosi University of Technology, Jolfa Avenue, Seyed Khandan, \\ P.O. Box 15875-4416, Tehran, Iran \\ ${ }^{2}$ Department of Mathematics and Applied Mathematics, University of Venda, Private Bag X5050, Thohoyandou 0950, South Africa
}

Correspondence should be addressed to S. Shateyi; stanford.shateyi@univen.ac.za

Received 2 December 2014; Accepted 13 February 2015

Academic Editor: Bing-Jean Lee

Copyright (C) 2015 S. Hatami et al. This is an open access article distributed under the Creative Commons Attribution License, which permits unrestricted use, distribution, and reproduction in any medium, provided the original work is properly cited.

We introduce some New Quadrature Formulas by using Jacoby polynomials and Laguerre polynomials. These formulas can be obtained for a finite and infinite interval and also separately for the even or odd order of derivatives. By using the properties of error functions of the above orthogonal polynomials we can obtain the error functions for these formulas. Application of the new approaches increases their precision degrees. Finally, some examples are given to illuminate the details.

\section{Introduction}

Let $m$ be a positive integer, $f^{(n)}(x)$ the derivative of order $n$ for the function $f(x), P_{k}(x)$ the set of polynomials of degree at most $k$, and $\mu(x)$ a positive and integrable function on the interval $[a, b]$ throughout this paper.

We consider the quadrature formula as follows:

$$
I(f ; \mu):=\int_{a}^{b} f(x) \mu(x) d x
$$

If $x_{1}, \ldots, x_{n}$ are chosen as the $n$ distinct zeros of orthogonal polynomial of degree $n$ in the family of orthogonal polynomials $[1,2]$ associated with $\mu(x)$, then the formula

$$
I(f ; \mu)=Q_{0}(f ; \mu):=\sum_{j=1}^{n} \lambda_{j} f\left(x_{j}\right)
$$

is exact for $P_{2 n-1}$. That is, the positive weights $\left\{\lambda_{i}\right\}_{i=1}^{n}$ are usually determined in a way that formula (2) is exact for the polynomials of degree as high as possible. Attempts to obtain similar quadrature formulas have not been restricted just to $f(x)$; many researchers began to obtain some New Quadrature Formulas based on $f(x)$ and its derivatives. For example, Turán was among the first who considered in his interesting paper in 1950 [3] the following quadrature rules:

$$
Q_{s}(f ; \mu):=\sum_{k=0}^{2 s} \sum_{j=1}^{n} \lambda_{k j} f^{(k)}\left(x_{j, s}\right) .
$$

He showed that these rules have maximum degree of precision as $2(s+1) n-1$. Turán's attempt on quadrature formulae attracted other researchers to expand this field. They began to follow his works and obtained several formulas [48]. The other case of quadrature formulae based on $f(x)$ and its derivatives is called Gaussian Birkhoff quadrature. For instance, Jetter [9] obtained new Gaussian quadrature formulas based on Birkhoff-type data. He used special cases of data as pyramidal type data in incidence matrices. He and Dyn [10] also worked on existence condition for these quadrature formulas that are the generalized form of the Gaussian quadrature. In another paper, he showed in [11] that the formulas introduced in [9] are unique. Bojanov and Nikolov [12] showed that the error of the quadrature formulas depends monotonically on the data. Wang and Guo [13] obtained the asymptotic estimate of nodes and weights of Gaussian-Lobatto-Legendre-Birkhoff quadrature formulas. They presented a user-oriented implementation of pesudospectral methods based on these quadrature nodes 
for Neumann problems. Milovanović and Đorđević [14] obtained nine-point quadrature formulae of interpolatory type of analytical functions. Varma [15] obtained a $(0,2)$ quadrature formula by using the fundamental polynomials

$$
\begin{aligned}
\int_{-1}^{1} f(x) d x= & \frac{3(f(1)+f(-1))}{n(2 n-1)} \\
& -\sum_{k=2}^{n-1} \frac{2(2 n-3) f\left(x_{k n}\right)}{n(n-2)(2 n-1) P_{n-1}^{2}} \\
& +\sum_{k=2}^{n-1} \frac{\left(1-x_{k n}^{2}\right) f^{\prime \prime}\left(x_{k n}\right)}{n(n-1)(n-2)(2 n-1) P_{n-1}^{2}\left(x_{k n}\right)},
\end{aligned}
$$

where $P_{n-1}(x)$ denotes the Legendre polynomials of degree $\leq n-1$ and $x_{k n}$ is the matrix incidence element in row $k$ and column $n$. In other paper [16], he obtained (4) with simple proof and did not use the fundamental polynomials. He [17] considered $(0, M, P)$ quadrature formulas where $M$ and $P$ are even positive integers. The nodes are $\theta_{k n}(=2 k \pi / n, k=$ $0,1, \ldots, n-1)$ and this formula is exact for all of trigonometric polynomials of order $3 n$ given by

$$
f(\theta)=\sum_{k=0}^{3 n-1}\left(a_{k} \cos k \theta+b_{k} \sin k \theta\right)+b_{3 n} \sin 3 n \theta
$$

Milovanovic and Varma [18] have given two types of $(0,3)$ and $(0,4)$ quadrature formulas. These quadrature formulas are exact for polynomials of degree at most $2 n-1$. Suzuki [19] considered a special type of incidence matrices $E=\left(e_{i j}\right)$ defined by $e_{i j}=0,1,(1 \leq i \leq 2,0 \leq j \leq n-1)$ and $\sum_{j=0}^{n-1} e_{1 j}+\sum_{j=0}^{n-1} e_{2 j}=n$ and obtained a special class of HermieBirkhoff quadrature as follows:

$$
\int_{a}^{b} P(x) d x=\sum_{e_{k j}} \omega_{k j} P^{(j)}\left(x_{i}\right)
$$

where (6) is exact for any polynomials $P(x)$ with degree at most $n-1$ and $\omega_{k j}$ s are weight-coefficient independent of $P(x)$. Lénárd [20] obtained a Birkhoff-type quadrature formula with Laguerre abscissas as follows:

$$
\begin{aligned}
\int_{0}^{+\infty} f(x) e^{-x} d x \sim & \sum_{j=0}^{n} A_{j} f\left(x_{j}\right)+\sum_{j=1}^{n} B_{j} f^{\prime}\left(x_{j}^{*}\right) \\
& +\sum_{j=0}^{k-1} C_{j} f^{(j)}(0),
\end{aligned}
$$

where $\left\{x_{j}\right\}_{j=1}^{n}$ and $\left\{x_{j}^{*}\right\}_{j=1}^{n}$ are the zeros of the Laguerre polynomials $L_{n}^{(k)}(x)$ and $L_{n}^{(k-1)}(x)$, respectively [1, 2]. Equation (7) is also exact for the polynomials of degree at most $2 n+k$.
She gives in another paper [21], a Birkhoff quadrature formula as follows:

$$
\begin{aligned}
\int_{-1}^{1} f(x) d x \approx & \sum_{i=0}^{n} A_{i} f^{\prime}\left(x_{i}\right)+\sum_{i=1}^{n-1} B_{i} f\left(x_{i}^{*}\right) \\
& +\sum_{j=0}^{k} C_{j}\left(f^{(j)}(1)+(-1)^{j} f^{(j)}(-1)\right),
\end{aligned}
$$

which is exact for the polynomials of degree $\leq 2 n+2 k+1$. She considered that the nodes $\left\{x_{i}\right\}_{i=1}^{n}$ and $\left\{x_{i}^{*}\right\}_{i=1}^{n-1}$ to solve (8) are the zeros of the ultraspherical polynomials $P_{n}^{(k)}(x)$ and $P_{n}^{(k)^{\prime}}(x)$, respectively.

Eslahchi and Dehghan [22] obtained two formulas as follows:

$$
\begin{aligned}
\int_{0}^{1} f(x) d x= & {\left[\sum_{i=0}^{m} \frac{(-1)^{k-1}}{k !} x^{k} f^{(k-1)}(x)\right]_{0}^{1} } \\
& +\sum_{i=1}^{n} w_{n i}^{(0, m)} f^{(n)} x_{n i}^{(0, m)}+f^{(n)} R_{n}^{(0, m)}, \\
\int_{0}^{1} e^{-x} f(x) d x= & {\left[-e^{-x} \sum_{i=0}^{m-1} f^{(k)}(x)\right]_{0}^{1} } \\
& +\sum_{i=1}^{n} w_{n k}^{(0)} f^{(m)} x_{n k}^{(0)}+R_{n}^{(0)} f^{(m)},
\end{aligned}
$$

where $\left\{x_{n i}^{(0, m)}\right\}_{i=0}^{n}$ and $x_{n k}^{(0)}$ are the roots of Jacobi Polynomials $P_{n}^{(0, m)}(x)$ and Laguerre Polynomials $L_{n}^{(0)}(x)$, respectively.

In this paper we intend to obtain some New Quadrature Formulas for the even or odd degree of derivatives separately. For instance, if we consider the even order of derivatives in the finite interval $[0,1]$, then we can obtain the formula as follows:

$$
\begin{aligned}
\int_{0}^{1} f(x) d x= & {\left[\sum_{i=0}^{m-1} \frac{x^{2 i+1}}{2^{i}(2 i+1) !} f^{(2 i)}(x)\right.} \\
& \left.-\sum_{i=1}^{m} \frac{x^{2 i}}{2^{i-1}(2 i) !} f^{(2 i-1)}(x)\right]_{0}^{1} \\
& +\sum_{i=1}^{m^{\prime}} \sum_{j=1}^{n-i} \frac{1}{2^{i}(2 i) !} \omega_{n-i, j}^{(0,2 i)} f^{(2 i)}\left(x_{n-i, j}^{(0,2 i)}\right) \\
& +\sum_{k=1}^{m^{\prime}} \frac{1}{2^{m} m !} R_{n-k}^{(0.2 k)}\left[f^{(2 k)}\right] .
\end{aligned}
$$

Also, if we consider the odd order of derivatives in the finite interval $[0,1]$ we get another quadrature formula. Replacing $[0,+\infty]$ instead $[0,1]$, we obtain two other formulas for the even or odd degree of derivatives.

The paper is organized as follows: in Section 2 we introduce Gauss-Jacobi quadrature and Gauss-Laguerre quadrature rules. In Section 3 we express the algorithms for obtaining New Quadrature Formulas and the error functions. Section 4 contain some examples for illumination and details. 


\section{Preliminaries}

In this section we introduce briefly the quadrature rules based on orthogonal polynomials. So, we consider the general form of weighted quadrature rules [23-26] which is defined as

$$
\begin{aligned}
\int_{a}^{b} f & (x) d \mu(x) \\
& =\sum_{i=1}^{n} \omega_{n, i} f\left(x_{n, i}\right)+\sum_{k=1}^{m} v_{m, k} f\left(\tau_{m, k}\right)+R_{n, m}[f],
\end{aligned}
$$

where $\left\{\omega_{n, i}\right\}_{i=1}^{n},\left\{v_{m, k}\right\}_{k=1}^{m}$ are unknown coefficients, $\left\{x_{n, i}\right\}_{i=1}^{n}$ unknown nodes, and $\left\{\tau_{m, k}\right\}_{k=1}^{m}$ the predetermined nodes. The error function [23-25], $R_{n, m}[f]$, can be determined by

$$
\begin{array}{r}
R_{n, m}[f] \\
=\frac{f^{(2 n+m)}(\xi)}{(2 n+m) !} \int_{a}^{b} \prod_{k=1}^{m}\left(x-\tau_{m, k}\right) \prod_{i=1}^{n}\left(x-x_{n, i}\right)^{2} d \mu(x), \\
a<\xi<b .
\end{array}
$$

For $m=0$ (11) changes to

$$
\int_{a}^{b} f(x) d \mu(x)=\sum_{i=1}^{n} \omega_{n, i} f\left(x_{n, i}\right)+R_{n, 0}[f] .
$$

It can be proved among the quadrature rules that the Gaussian quadrature (for $m=0$ in (13)) has the highest precision degree $(2 n-1)$. In other words

$$
R_{n, 0}[f]=0 \Longleftrightarrow f \in P_{2 n-1} .
$$

Obviously the nodes $\left\{x_{n, i}\right\}_{i=1}^{n}$ are the zeros of a sequence of polynomials $P_{n}(x)$, which are orthogonal [2] with respect to $d \mu(x)$ on $[a, b]$; that is,

$$
\int_{a}^{b} P_{n}(x) P_{m}(x) d \mu(x)=\left(\int_{a}^{b} P_{n}^{2}(x) d \mu(x)\right) \delta_{m, n},
$$

where $\delta_{m, n}$ is the Kronecker delta. Moreover, we simply can compute the coefficients $\left\{\omega_{n, i}\right\}_{i=1}^{n}$ by using the following formula [27]:

$$
\omega_{n, i}=\frac{\left\langle P_{n-1}(x), P_{n-1}(x)\right\rangle}{P_{n-1}\left(x_{n, i}\right) P_{n-1}^{\prime}\left(x_{n, i}\right)}, \quad i=1,2, \ldots, n
$$

where

$$
\left\langle P_{n}(x), P_{m}(x)\right\rangle=\int_{a}^{b} P_{n}(x) P_{m}(x) d \mu(x) .
$$

2.1. Gauss-Jacobi Quadrature. Let us consider $d \mu(x)=(1-$ $x)^{\alpha} x^{\beta} d x$ (Jacobi weight function) with $\alpha, \beta>-1$ on $[0,1]$, so, (13) can be written as

$$
\int_{0}^{1}(1-x)^{\alpha} x^{\beta} f(x) d x=\sum_{i=1}^{n} \omega_{n, i}^{(\alpha, \beta)} f\left(x_{n, i}^{(\alpha, \beta)}\right)+R_{n}^{(\alpha, \beta)},
$$

where $\left\{x_{n, i}^{(\alpha, \beta)}\right\}_{i=1}^{n}$ are the zeros of Jacobi polynomials on $[0,1]$ which are defined [28] by

$$
\begin{aligned}
& P_{n}^{(\alpha, \beta)}(x)= \sum_{i=0}^{n}(-1)^{n-i}\left(\begin{array}{c}
\alpha+n \\
i
\end{array}\right)\left(\begin{array}{c}
\beta+n \\
n-i
\end{array}\right)(1-x)^{n-i} x^{i}, \\
& x \in[0,1], \\
& \omega_{n, i}^{(\alpha, \beta)}=\frac{2^{\alpha+\beta+1} \Gamma(\alpha+n+1) \Gamma(\beta+n+1)}{n ! \Gamma(\alpha+\beta+n+1)\left(1-\left(2 x_{n, i}^{(\alpha, \beta)}-1\right)^{2}\right)} \\
& \cdot\left(\left.\frac{d}{d x} P_{n}^{(\alpha, \beta)}(x)\right|_{x=x_{n, i}^{(\alpha, \beta)}}\right)^{-2},
\end{aligned}
$$

where $i=1,2, \ldots, n$. Furthermore if $f(x)$ has a continuous derivative of order $2 n$ on $[0,1]$ then the reminder term of formula (13) is given as

$$
\begin{aligned}
& R_{n}^{(\alpha, \beta)}(f) \\
&= \frac{f^{(2 n)}(\eta)}{(2 n) !}\left(\frac{n ! \Gamma(\alpha+\beta+n+1)}{\Gamma(\alpha+\beta+2 n+1)}\right)^{2} \\
& \cdot \int_{0}^{1}(1-x)^{\alpha} x^{\beta}\left(P_{n}^{(\alpha, \beta)}(x)\right)^{2} d x \\
&= \frac{f^{(2 n)}(\eta)}{(2 n) !} \frac{n ! \Gamma(\alpha+n+1) \Gamma(\beta+n+1) \Gamma(\alpha+\beta+n+1)}{(\alpha+\beta+2 n+1)(\Gamma(\alpha+\beta+2 n+1))^{2}}, \\
& 0<\eta<1 .
\end{aligned}
$$

2.2. Gauss-Laguerre Quadrature. Another class of quadrature rules that is applicable in this paper is Gauss-Laguerre quadrature that can be obtained by selecting the interval of quadrature $[0,+\infty]$ and $d \mu(x)=x^{s} e^{-x} d x,(s>-1)$ in (13). So, we have

$$
\int_{0}^{+\infty}(x)^{s} e^{-x} f(x) d x=\sum_{i=1}^{n} \omega_{n, i}^{(s)} f\left(x_{n, i}^{(s)}\right)+R_{n}^{(s)}[f],
$$

where $\left\{x_{n, i}^{(s)}\right\}_{i=1}^{n}$ are the zeros of Laguerre polynomials [28] of the form

$$
L_{n}^{(s)}(x)=\sum_{i=0}^{n}\left(\begin{array}{l}
n+s \\
n-i
\end{array}\right) \frac{(-x)^{i}}{i !}, \quad x \in[0,+\infty),
$$

and the weight functions are as follows:

$$
\omega_{n, i}^{(s)}=\frac{\Gamma(s+n+1)}{n ! x_{n, i}^{(s)}}\left(\left.\frac{d}{d x} L_{n}^{(s)}(x)\right|_{x=x_{n, i}^{(s)}}\right)^{-2}, \quad i=1,2, \ldots, n,
$$


and error of this quadrature formula is

$$
\begin{aligned}
R_{n}^{(s)}[f] & =\frac{f^{(2 n)}(\eta)}{(2 n) !}(n !)^{2} \int_{0}^{+\infty} x^{s} e^{-x}\left(L_{n}^{(s)}(x)\right)^{2} d x \\
& =\frac{f^{(2 n)}(\eta)}{(2 n) !}(n !)^{2} \Gamma(n+s+1), \quad 0<\eta<+\infty .
\end{aligned}
$$

Also if $s=0$ in (21), it is called Gauss-Laguerre quadrature formulas.

\section{New Quadrature Formulas}

3.1. New Quadrature Formulas in Finite Interval. In this section we obtain two New Quadrature Formulas. It should be noted that we must use the even or odd degree of derivatives separately. By using error function (20) for Jacobi quadrature formulas we can obtain the error functions for these quadrature formulas. Error functions can also be obtained by a similar process for the error of composed trapezoidal rules [23, page 129]. We first consider the even order of derivatives and because of similarity ignore the odd degree of derivatives. Now, we have

$$
\int_{a}^{b} u d v=\left.u v\right|_{a} ^{b}-\int_{a}^{b} v d u
$$

such that

$$
\begin{gathered}
d v=d x, \quad v=x, \\
u=f(x),
\end{gathered} \quad \begin{gathered}
v u=f^{\prime}(x) d x, x \in[0,1] ;
\end{gathered}
$$

then we have

$$
\int_{0}^{1} f(x) d x=\left.x f(x)\right|_{0} ^{1}-\int_{0}^{1} x f^{\prime}(x) d x
$$

Now, consider the integral in the right-hand side of (27) and use the following change of variable:

$$
\begin{gathered}
d v=x d x, \\
u=f^{\prime}(x),
\end{gathered} \begin{gathered}
v=\frac{1}{2} x^{2}, \\
d u=f^{\prime \prime}(x) d x, x \in[0,1],
\end{gathered}
$$

which gives

$$
\int_{0}^{1} x f^{\prime}(x) d x=\left.\frac{1}{2} x^{2} f^{\prime}(x)\right|_{0} ^{1}-\frac{1}{2} \int_{0}^{1} x^{2} f^{\prime \prime}(x) d x .
$$

Substituting (29) into (27) gives

$$
\begin{aligned}
\int_{0}^{1} f(x) d x= & {\left.\left[x f(x)-\frac{1}{2} x^{2} f^{\prime}(x)\right]\right|_{0} ^{1} } \\
& +\frac{1}{2} \int_{0}^{1}\left(\frac{1}{2} x^{2}+\frac{1}{2} x^{2}\right) f^{\prime \prime}(x) d x \\
= & {\left.\left[x f(x)-\frac{1}{2} x^{2} f^{\prime}(x)\right]\right|_{0} ^{1} } \\
& +\frac{1}{4} \int_{0}^{1} x^{2} f^{\prime \prime}(x) d x+\frac{1}{4} \int_{0}^{1} x^{2} f^{\prime \prime}(x) d x .
\end{aligned}
$$

If we apply the Gauss-Jacobi quadrature rules with respect to the weight function $x^{2}$ on $[0,1]$ for $f^{\prime \prime}(x)$ (instead of $f(x)$ in (18)), we obtain the following new relation:

$$
\int_{0}^{1} x^{2} f^{\prime \prime}(x) d x=\sum_{i=1}^{n-1} \omega_{n-1, i}^{(0,2)} f^{\prime \prime}\left(x_{n-1, i}^{(0,2)}\right)+R_{n-1}^{(0,2)}\left[f^{\prime \prime}\right]
$$

where $\left\{x_{n-1, i}^{(0,2)}, \omega_{n-1, i}^{(0,2)}\right\}_{i=1}^{n-1}$ and $R_{n-1}^{(0,2)}\left[f^{\prime \prime}\right]$ are previously defined in ((19)-(20)). The degree of the error function in (31) is $2 n$. Now, if we put

$$
\begin{gathered}
d v=x^{2} d x, \\
u=f^{\prime \prime}(x),
\end{gathered} \begin{gathered}
v=\frac{1}{3} x^{3}, \\
d u=f^{(3)}(x) d x, \quad x \in[0,1],
\end{gathered}
$$

we have

$$
\int_{0}^{1} x^{2} f^{\prime \prime}(x) d x=\left.\frac{1}{3} x^{3} f^{\prime \prime}(x)\right|_{0} ^{1}-\frac{1}{3} \int_{0}^{1} x^{3} f^{(3)}(x) d x ;
$$

by substituting ((31), (33)) in (30) again, we get

$$
\begin{aligned}
\int_{0}^{1} f(x) d x= & {\left.\left[x f(x)-\frac{1}{2} x^{2} f^{\prime}(x)+\frac{1}{3 \times 4} x^{3} f^{\prime \prime}(x)\right]\right|_{0} ^{1} } \\
& +\frac{1}{4} \sum_{i=1}^{n-1} \omega_{n-1, i}^{(0,2)} f^{\prime \prime}\left(x_{n-1, i}^{(0,2)}\right)+\frac{1}{4} R_{n-1}^{(0,2)}\left[f^{\prime \prime}\right] \\
& -\frac{1}{3 \times 4} \int_{0}^{1} x^{3} f^{(3)}(x) d x .
\end{aligned}
$$

Here, we consider integration in the right-hand side of (34) and we use again the following change of variable:

$$
\begin{aligned}
& d v=x^{3} d x, \quad \Longleftrightarrow \quad v=\frac{1}{4} x^{4}, \\
& u=f^{(3)}(x), \Longleftrightarrow d u=f^{(4)}(x) d x, x \in[0,1] \text {; }
\end{aligned}
$$

then we can write

$$
\int_{0}^{1} x^{3} f^{(3)}(x) d x=\left.\frac{1}{4} x^{4} f^{(3)}(x)\right|_{0} ^{1}-\frac{1}{4} \int_{0}^{1} x^{4} f^{(4)}(x) d x
$$

substituting (36) into (34), then we get

$$
\begin{aligned}
\int_{0}^{1} f(x) d x= & {\left[x f(x)-\frac{1}{2} x^{2} f^{\prime}(x)+\frac{1}{3 \times 4} x^{3} f^{\prime \prime}(x)\right.} \\
& \left.-\frac{1}{2 \times 4 !} x^{4} f^{(3)}(x)\right]\left.\right|_{0} ^{1} \\
& +\frac{1}{4} \sum_{i=1}^{n-1} \omega_{n-1, i}^{(0,2)} f^{\prime \prime}\left(x_{n-1, i}^{(0,2)}\right)+\frac{1}{4} R_{n-1}^{(0,2)}\left[f^{\prime \prime}\right] \\
& +\frac{1}{2 \times 4 !} \int_{0}^{1} x^{4} f^{(4)}(x) d x .
\end{aligned}
$$


Now, we consider ((38)-(39)) and substitute these formulas into (37). Consider

$$
\begin{gathered}
\int_{0}^{1} x^{4} f^{(4)}(x) d x=\frac{1}{2} \int_{0}^{1} x^{4} f^{(4)}(x) d x+\frac{1}{2} \int_{0}^{1} x^{4} f^{(4)}(x) d x \\
\int_{0}^{1} x^{4} f^{(4)}(x) d x=\sum_{i=1}^{n-2} \omega_{n-2, i}^{(0,4)} f^{(4)}\left(x_{n-2, i}^{(0,4)}\right)+R_{n-2}^{(0,4)}\left[f^{(4)}\right] .
\end{gathered}
$$

Therefore, we have

$$
\begin{aligned}
\int_{0}^{1} f(x) d x= & {\left[x f(x)-\frac{1}{2} x^{2} f^{\prime}(x)+\frac{1}{3 \times 4} x^{3} f^{\prime \prime}(x)\right.} \\
& \left.-\frac{1}{2 \times 4 !} x^{4} f^{(3)}(x)\right]\left.\right|_{0} ^{1} \\
& +\frac{1}{4} \sum_{i=1}^{n-1} \omega_{n-1, i}^{(0,2)} f^{\prime \prime}\left(x_{n-1, i}^{(0,2)}\right) \\
& +\frac{1}{4 \times 4 !} \sum_{i=1}^{n-2} \omega_{n-2, i}^{(0,4)} f^{(4)}\left(x_{n-2, i}^{(0,4)}\right) \\
& +\frac{1}{4} R_{n-1}^{(0,2)}\left[f^{\prime \prime}\right]+\frac{1}{4 \times 4 !} R_{n-2}^{(0,4)}\left[f^{(4)}\right] \\
& +\frac{1}{4 \times 4 !} \int_{0}^{1} x^{4} f^{(4)}(x) d x .
\end{aligned}
$$

It is worthy of attention that the degree of error function in (39) is $2 n$. If we apply this process only for the even degree of derivatives, then for 6 th order of derivatives we can obtain the relation as follows:

$$
\begin{aligned}
& \int_{0}^{1} f(x) d x=\left[x f(x)-\frac{1}{2} x^{2} f^{\prime}(x)\right. \\
&\left.+\ldots+\frac{1}{4 \times 6 !} x^{6} f^{(5)}(x)\right]\left.\right|_{0} ^{1} \\
&+\frac{1}{4} \sum_{i=1}^{n-1} \omega_{n-1, i}^{(0,2)} f^{\prime \prime}\left(x_{n-1, i}^{(0,2)}\right) \\
&+\frac{1}{4 \times 4 !} \sum_{i=1}^{n-2} \omega_{n-2, i}^{(0,4)} f^{(4)}\left(x_{n-2, i}^{(0,4)}\right) \\
&+\frac{1}{4} R_{n-1}^{(0,2)}\left[f^{\prime \prime}\right]+\frac{1}{4 \times 4 !} R_{n-2}^{(0,4)}\left[f^{(4)}\right] \\
&+\frac{1}{4 \times 6 !} \int_{0}^{1} x^{6} f^{(6)}(x) d x .
\end{aligned}
$$

By iterating this process and using the even order of derivatives we write the quadrature formulae as follows:

$$
\begin{aligned}
\int_{0}^{1} f(x) d x= & {\left[\sum_{i=0}^{m-1} \frac{x^{2 i+1}}{2^{i}(2 i+1) !} f^{(2 i)}(x)\right.} \\
& \left.-\sum_{i=1}^{m} \frac{x^{2 i}}{2^{i-1}(2 i) !} f^{(2 i-1)}(x)\right]\left.\right|_{0} ^{1} \\
& +\sum_{i=1}^{m^{\prime}} \sum_{j=1}^{n-i} \frac{1}{2^{i}(2 i) !} \omega_{n-i, j}^{(0,2 i)} f^{(2 i)}\left(x_{n-i, j}^{(0,2 i)}\right) \\
& +\sum_{k=1}^{m^{\prime}} \frac{1}{2^{m} m !} R_{n-k}^{(0,2 k)}\left[f^{(2 k)}\right]
\end{aligned}
$$

where $\sum^{\prime}$ shows twice the coefficient of last part of summation. The precision degree in this formula is $2 m n-m(m+1)-1$, which indicates the advantages of the new approach. Now, we consider the error functions for this formula:

$$
\begin{aligned}
R_{n-k}^{(0,2 k)}\left[f^{(2 k)}\right] \\
=\frac{f^{(2 n)}\left(\lambda_{k}\right)}{(2 n-2 k) !} \\
\quad \cdot \frac{(n-k) ! \Gamma(n-k+1) \Gamma(n+k+1) \Gamma(n+k+1)}{(2 n+1)(\Gamma(2 n+1))^{2}} \\
=\theta_{k} f^{(2 n)}\left(\lambda_{k}\right), \quad 1 \leq k \leq n .
\end{aligned}
$$

For simplifying the error term of (42) from $m$ points, we consider the error functions for composed trapezoidal rules and we will obtain a relation that is similar to the relation built in [23, page 129]

$$
\min _{i} f^{(2 n)}\left(\lambda_{i}\right) \leq \frac{1}{m} \sum_{k=1}^{m} f^{(2 n)}\left(\lambda_{k}\right) \leq \max _{i} f^{(2 n)}\left(\lambda_{i}\right) .
$$

Let us consider the following relations:

$$
\begin{gathered}
\theta_{1} \min _{i} f^{(2 n)}\left(\lambda_{i}\right) \leq \theta_{1} f^{(2 n)}\left(\lambda_{1}\right) \leq \theta_{1} \max _{i} f^{(2 n)}\left(\lambda_{i}\right), \\
\theta_{2} \min _{i} f^{(2 n)}\left(\lambda_{i}\right) \leq \theta_{2} f^{(2 n)}\left(\lambda_{2}\right) \leq \theta_{2} \max _{i} f^{(2 n)}\left(\lambda_{i}\right), \\
\vdots \\
\theta_{m} \min _{i} f^{(2 n)}\left(\lambda_{i}\right) \leq \theta_{m} f^{(2 n)}\left(\lambda_{m}\right) \leq \theta_{m} \max _{i} f^{(2 n)}\left(\lambda_{i}\right) .
\end{gathered}
$$
gives

Adding the left sides and right sides of (46), respectively,

$$
\min _{i} f^{(2 n)}\left(\lambda_{i}\right) \sum_{k=1}^{m} \theta_{k} \leq \sum_{i=1}^{m} \theta_{k} f^{(2 n)}\left(\lambda_{k}\right) \leq \max _{i} f^{(2 n)}\left(\lambda_{i}\right) \sum_{k=1}^{m} \theta_{k} .
$$


Finally, we have

$$
\min _{i} f^{(2 n)}\left(\lambda_{i}\right) \leq \frac{\sum_{i=1}^{m} \theta_{k} f^{(2 n)}\left(\lambda_{k}\right)}{\sum_{k=1}^{m} \theta_{k}} \leq \max _{i} f^{(2 n)}\left(\lambda_{i}\right) .
$$

Now, we suppose $f^{(2 n)}(x)$ is continuous; there exist a $\lambda \epsilon$ $\left[\min _{i} \lambda_{i}, \max _{i} \lambda_{i}\right] \subset(0,1)$ such that $[23$, page 129$]$

$$
f^{(2 n)}(\lambda)=\frac{\sum_{i=1}^{m} \theta_{k} f^{(2 n)}\left(\lambda_{k}\right)}{\sum_{k=1}^{m} \theta_{k}}
$$

Thus, we have

$$
\sum_{k=1}^{m} R_{n-k}^{(0,2 k)}\left[f^{(2 k)}\right]=\sum_{k=1}^{m} \theta_{k} f^{(2 n)}\left(\lambda_{k}\right)=\sum_{k=1}^{m} \theta_{k} f^{(2 n)}(\lambda) .
$$

Apply the process for the odd order of derivatives gives another Quadrature Formula:

$$
\begin{aligned}
\int_{0}^{1} f(x) d x= & {\left[\sum_{i=0}^{m-1} \frac{x^{2 i+1}}{2^{i}(2 i+1) !} f^{(2 i)}(x)\right.} \\
& \left.-\sum_{i=1}^{m-1} \frac{x^{2 i}}{2^{i}(2 i-1) !} f^{(2 i-1)}(x)\right]\left.\right|_{0} ^{1} \\
& -\sum_{i=1}^{m^{\prime}} \sum_{j=1}^{n-i} \frac{1}{2^{i}(2 i-1) !} \omega_{n-i, j}^{(0,2 i-1)} f^{(2 i-1)}\left(x_{n-i, j}^{(0,2 i-1)}\right) \\
& -\sum_{k=1}^{m^{\prime}} \frac{R_{n-k}^{(0,2 k-1)}\left[f^{(2 k-1)}\right]}{2^{m}(m+1) !}
\end{aligned}
$$

Similar to the even degree of derivatives we can obtain the error function for the odd degree of derivatives with the precision degree $2 m n-m(m+1)-1$.

3.2. New Quadrature Formulas in Infinite Interval. Let us extend the results to infinite interval $[0,+\infty)$. In this section we also use the even or odd degree of derivatives similar to the pervious section and try to obtain error function for these formulas. We follow the same procedure in the previous section:

$$
\begin{gathered}
d v=e^{-1} d x, \\
u=f(x),
\end{gathered} \begin{gathered}
v=-e^{-x}, \\
d u=f^{\prime}(x) d x, \quad x \in[0,+\infty] ;
\end{gathered}
$$

then we have

$$
\int_{0}^{+\infty} e^{-x} f(x) d x=-\left.e^{-x} f(x)\right|_{0} ^{+\infty}+\int_{0}^{+\infty} e^{-x} f^{\prime}(x) d x
$$

The Gauss-Laguerre quadrature rules can be shown:

$$
\int_{0}^{+\infty} e^{-x} f^{(m)}(x) d x=\sum_{k=1}^{n} \omega_{n, k}^{(0)} f^{(m)}\left(x_{n, k}^{(0)}\right)+R_{n}^{(0)}\left[f^{(m)}\right],
$$

where $\left\{\omega_{n, k}^{(0)}\right\}_{k=1}^{n}$ are obtained from (23) and $\left\{x_{n, k}^{(0)}\right\}_{k=1}^{n}$ are the roots of Laguerre polynomials $L_{n}^{(0)}(x)$ and the error function is

$$
R_{n}^{(0)}\left[f^{(m)}\right]=\frac{f^{(2 n+m)}(\eta)}{(2 n) !}(n !)^{2} ; \quad 0<\eta<+\infty .
$$

Now, if we consider the even degree of derivatives and use the process for New Quadrature Formulas similar to the even order of derivatives in finite intervals (previous part) we can obtain another formula for quadrature formulae as follows:

$$
\begin{aligned}
\int_{0}^{+\infty} & e^{-x} f(x) d x \\
= & -\left.e^{-x}\left[\sum_{i=0}^{m-1} \frac{1}{2^{i}} f^{(2 i)}(x)+\sum_{i=1}^{m} \frac{1}{2^{i-1}} f^{(2 i-1)}(x)\right]\right|_{0} ^{+\infty} \\
& +\sum_{i=1}^{m^{\prime}} \frac{1}{2^{i}} \sum_{j=1}^{n-i} \omega_{n-i, j}^{(0)} f^{(2 i)}\left(x_{n-i, j}^{(0)}\right)+\sum_{k=1}^{m^{\prime}} \frac{1}{2^{k}} R_{n-k}^{(0)}\left[f^{(2 k)}\right],
\end{aligned}
$$

and if we consider the odd degree of derivatives we have

$$
\begin{aligned}
\int_{0}^{+\infty} & e^{-x} f(x) d x \\
= & -\left.e^{-x}\left[\sum_{i=0}^{m-1} \frac{1}{2^{i}} f^{(2 i)}(x)+\sum_{i=1}^{m-1} \frac{1}{2^{i-1}} f^{(2 i-1)}(x)\right]\right|_{0} ^{+\infty} \\
& +\sum_{i=1}^{m^{\prime}} \sum_{j=1}^{n-i} \frac{1}{2^{i}} \omega_{n-i, j}^{(0)} f^{(2 i-1)}\left(x_{n-i, j}^{(0)}\right) \\
& +\sum_{k=1}^{m^{\prime}} \frac{1}{2^{k}} R_{n-k}^{(0)}\left[f^{(2 k-1)}\right]
\end{aligned}
$$

To obtain a formula for the error function considering (55) and using (54) gives

$$
\begin{array}{r}
k=1, \quad R_{n-1}^{(0)}\left[f^{(2)}\right]=\frac{f^{(2 n)}\left(\lambda_{1}\right)}{(2 n-2) !}((n-1) !)^{2}=\theta_{1} f^{(2 n)}\left(\lambda_{1}\right), \\
0<\lambda_{1}<+\infty, \\
k=2, \quad R_{n-2}^{(0)}\left[f^{(4)}\right]=\frac{f^{(2 n)}\left(\lambda_{2}\right)}{(2 n-4) !}((n-2) !)^{2}=\theta_{2} f^{(2 n)}\left(\lambda_{2}\right),
\end{array}
$$$$
0<\lambda_{2}<+\infty
$$

and for $k=m$ we have

$$
\begin{aligned}
R_{n-m}^{(0)}\left[f^{(2 m)}\right] & =\frac{f^{(2 n)}\left(\lambda_{m}\right)}{(2 n-2 m) !}((n-m) !)^{2} \\
& =\theta_{m} f^{(2 n)}\left(\lambda_{m}\right), \quad 0<\lambda_{m}<+\infty .
\end{aligned}
$$


TABLE 1: $(0,2)$ quadrature formulae.

\begin{tabular}{lcr}
\hline$n$ & $A\left(G-J^{(0,2)}(n)\right)$ & $E_{J(0,2)}$ \\
\hline 1 & 0.3798784407 & $0.705 e-05$ \\
2 & 0.3798802883 & $0.520 e-05$ \\
3 & 0.3798852701 & $0.223 e-06$ \\
4 & 0.3798859533 & $0.460 e-06$ \\
5 & 0.3798855961 & $0.103 e-06$ \\
6 & 0.3798854710 & $0.220 e-07$ \\
7 & 0.3798854803 & $0.128 e-07$ \\
8 & 0.3798854927 & $0.378 e-08$ \\
9 & 0.3798854941 & $0.102 e-08$ \\
\hline
\end{tabular}

TABLE $2:(0,2,4)$ and $(0,2,4,6)$ quadrature formulas.

\begin{tabular}{ccccc}
\hline$n$ & $A\left(G-J^{(0,2,4)}(n)\right)$ & $E_{J(0,2,4)}$ & $A\left(G-J^{(0,2,4,6)}(n)\right)$ & $E_{J(0,2,4,6)}$ \\
\hline 2 & 0.3798983625 & $0.181 e-4$ & & $5.791 e-7$ \\
3 & 0.3798805451 & $2.567 e-7$ & 0.3798808675 & $6.930 e-9$ \\
4 & 0.3798802910 & $2.640 e-9$ & 0.3798802953 & $4.420 e-11$ \\
5 & 0.3798802883 & $1.982 e-11$ & 0.379880288 & $3.090 e-13$ \\
6 & 0.3798802883 & $8.250 e-14$ & 0.379880288 & $1.250 e-14$ \\
7 & 0.3798802883 & $3.000 e-15$ & 0.379880288 & $2.250 e-15$ \\
8 & 0.3798802883 & $1.500 e-15$ & 0.379880288 & \\
9 & 0.3798802883 & $1.500 e-15$ & & \\
\hline
\end{tabular}

TABLE 3: $(0,2)$ and $(0,2,4)$ quadrature formulas.

\begin{tabular}{lcccc}
\hline$n$ & $A\left(G-L_{(0,2)}^{(0)}(n)\right)$ & $E_{L(0,2)}$ & $A\left(G-L_{(0,2,4)}^{(0)}(n)\right)$ & $E_{L(0,2,4)}$ \\
\hline 1 & 0.4133137157 & $0.496 e-5$ & & \\
2 & 0.4133121416 & $0.338 e-5$ & 0.4133131291 & $0.437 e-5$ \\
3 & 0.4133097907 & $0.103 e-5$ & 0.4133087904 & $3.173 e-8$ \\
4 & 0.4133088464 & $8.770 e-8$ & 0.4133087681 & $9.369 e-9$ \\
5 & 0.4133086998 & $5.891 e-8$ & 0.4133087595 & $8.148 e-10$ \\
6 & 0.4133087434 & $1.533 e-8$ & 0.4133087588 & $5.866 e-11$ \\
7 & 0.4133087622 & $3.487 e-9$ & 0.4133087587 & $4.083 e-12$ \\
8 & 0.4133087643 & $5.560 e-9$ & 0.4133087587 & $3.227 e-13$ \\
\hline
\end{tabular}

Again, we consider previous part for calculating the error function in finite intervals.

\section{Numerical Examples}

In this section we want to present two examples to clarify the details. We use the notations and examples introduced in [22].

Let

$A\left(G-J^{\left(\alpha=0, \beta_{1}=m, \beta_{2}=p\right)}=n\right.$-points quadrature formulas with parameters $\left(\alpha, \beta_{1}, \beta_{2}\right)$ for $\{0, m, p\}$-order derivatives, respectively, in finite intervals;

$A\left(G-L_{(0, m, p)}^{(0)}(n)\right)=n$-points quadrature formulas with parameter 0 for $\{0, m, p\}$-order derivatives in infinite intervals;

$E_{J(L)(0, m, p)}=\mid$ Exact value - Approximation value $\mid$.
Example 1. As the first example for quadrature formulae in $[0,1]$, we use the following integral:

$$
A:=\int_{0}^{1} \frac{1}{1+e^{x}} d x=0.3798854930 .
$$

This example is computed for $(0,2),(0,2,4)$, and $(0,2,4,6)$ quadrature formulas by using $(42)$ for $f(x)=1 /(1+$ $\left.e^{x}\right)$ and the results are obtained and tabulated in Tables 1 and 2.

Example 2. For infinite interval $[0,+\infty),(55)$ is considered for computing

$$
A:=\int_{0}^{+\infty} e^{-x} e^{(\sin (x / 10)-\cos (x / 10))} d x=0.4133087587
$$

where $f(x)=e^{\sin (x / 10)-\cos (x / 10)}$.

This example is also computed for $(0,2),(0,2,4)$, and $(0,2,4,6)$ quadrature formulas and the results tabulated in Tables 3 and 4 . 
TABLE 4: $(0,2,4,6)$ quadrature formulae.

\begin{tabular}{ccc}
\hline$n$ & $A\left(G-L_{(0,246)}^{(0)}(n)\right)$ & $E_{L(0,2,4,6)}$ \\
\hline 3 & 0.4133090947 & $3.359 e-7$ \\
4 & 0.4133087725 & $1.381 e-8$ \\
5 & 0.4133087597 & $1.026 e-9$ \\
6 & 0.4133087588 & $5.828 e-11$ \\
7 & 0.4133087587 & $5.269 e-12$ \\
8 & 0.4133087587 & $6.423 e-13$ \\
\hline
\end{tabular}

\section{Conclusion}

In this paper we introduced some New Quadrature Formulas by using Jacoby polynomials and Laguerre polynomials for a finite and infinite interval and also separately for the even or odd order of derivatives. The error functions of the above orthogonal polynomials are obtained for these formulas. The precision degrees are increased.

\section{Conflict of Interests}

The authors declare that there is no conflict of interests regarding the publication of this paper.

\section{References}

[1] W. Gautschi, Orthogonal Polynomials, Oxford University Press, 2004.

[2] T. S. Chihara, An Introduction to Orthogonal Polynomials, Gordon and Breach Science, New York, NY, USA, 1978.

[3] P. Turán, "On the theory of the mechanical quadrature," Acta Universitatis Szegediensis, vol. 12, pp. 30-37, 1950.

[4] M. M. Spalević, "Calculation of Chakalov-Popoviciu quadratures of Radau and Lobatto type," The ANZIAM Journal, vol. 43, no. 3, pp. 429-447, 2002.

[5] G. V. Milovanovic and M. M. Spalevic, "Gauss-Turán quadratures of Kronrod type for generalized Chebyshev weight functions," Calcolo, vol. 43, no. 3, pp. 171-195, 2006.

[6] Y. G. Shi, "On Turán quadrature formulas for the Chebyshev weight," Journal of Approximation Theory, vol. 96, no. 1, pp. 101110, 1999.

[7] Y. G. Shi, “Turán quadrature formulas and christoffel type functions for the chebyshev polynomials of the second kind," Acta Mathematica Hungarica, vol. 85, no. 3, pp. 253-263, 1999.

[8] L. Gori and C. A. Micchelli, "On weight functions which admit explicit Gauss-Turan quadrature formulas," Mathematics of Computation, vol. 65, no. 216, pp. 1567-1581, 1996.

[9] K. Jetter, "A new class of Gaussian quadrature formulas based on Birkhoff type data," SIAM Journal on Numerical Analysis, vol. 19, no. 5, pp. 1081-1089, 1982.

[10] N. Dyn and K. Jetter, "Existence of Gaussian quadrature formulas for Birkhoff type data," Archiv der Mathematik, vol. 52, no. 6, pp. 588-594, 1989.

[11] K. Jetter, "Uniqueness of Gauss-Birkhoff quadrature formulas," SIAM Journal on Numerical Analysis, vol. 24, no. 1, pp. 147-154, 1987.

[12] B. Bojanov and G. Nikolov, "Comparison of Birkhoff type quadrature formulae," Mathematics of Computation, vol. 54, no. 190, pp. 627-648, 1990.
[13] L. L. Wang and B. Y. Guo, "Interpolation approximations based on Gauss-Lobatto-LEGendre-Birkhoff quadrature," Journal of Approximation Theory, vol. 161, no. 1, pp. 142-173, 2009.

[14] G. V. Milovanović and R. Ž. Đorđević, "On a generalization of modified Birkhoff-Young quadrature formula," Univerzitet $u$ Beogradu, Publikacije Elektrotehniv ckog Fakulteta, pp. 130-134, 1982.

[15] A. K. Varma, "On some open problems of P. Turán concerning Birkhoff interpolation," Transactions of the American Mathematical Society, vol. 274, no. 2, pp. 797-808, 1982.

[16] A. K. Varma, "On Birkhoff quadrature formulas," Proceedings of the American Mathematical Society, vol. 97, no. 1, pp. 38-40, 1986.

[17] A. K. Varma, "On Birkhoff quadrature formulas II," Acta Mathematica Hungarica, vol. 62, no. 1-2, pp. 15-19, 1993.

[18] G. V. Milovanovic and A. K. Varma, "On Birkhoff $(0,3)$ and $(0,4)$ quadrature formula," Numerical Functional Analysis and Optimization, vol. 18, no. 3-4, pp. 427-433, 1997.

[19] C. Suzuki, "Two-point Hermite-Birkhoff quadrature and its applications to numerical solution of ODE," in Numerical Analysis of Ordinary Differential Equations and Its Applications, vol. 18, pp. 43-57, 1997.

[20] M. Lénárd, "PAL-type interpolation and quadrature formulae on Laguerre abscissas," Mathematica Pannonica, vol. 15, no. 2, pp. 265-274, 2004.

[21] M. Lénárd, "Birkhoff quadrature formulae based on the zeros of jacobi polynomials," Mathematical and Computer Modelling, vol. 38, no. 7-9, pp. 917-927, 2003.

[22] M. R. Eslahchi and M. Dehghan, "Quadrature rules using an arbitrary fixed order of derivatives," Computers \& Mathematics with Applications, vol. 57, no. 7, pp. 1212-1225, 2009.

[23] J. Stoer and P. Bulirsch, Introduaction to Numerical Analysis, Springer, New York, NY, USA, 2nd edition, 1993.

[24] V. I. Krylov, Approximate Calculation of Integrals, Macmillan, New York, NY, USA, 1962.

[25] R. Davis and P. Rabinowitz, Methods of Numerical Integration, Computer Science and Applied Mathematics, Academic Press, New York, NY, USA, 2nd edition, 1984.

[26] G. M. Phillips, Interpolation and Approximation by Polynomials, Springer, New York, NY, USA, 2003.

[27] W. H. Press, S. A. Teukolsky, W. T. Vetterling, and B. P. Flannery, Numerical Recipes in Fortran: The Art of Scientific Computing, Cambridge University Press, Cambridge, UK, 2nd edition, 1992.

[28] A. Ghizzetti and A. Ossicini, Quadrature Formulae, Academic Press, New York, NY, USA, 1970. 


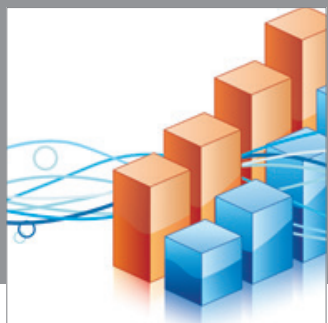

Advances in

Operations Research

mansans

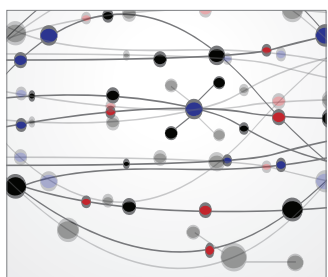

The Scientific World Journal
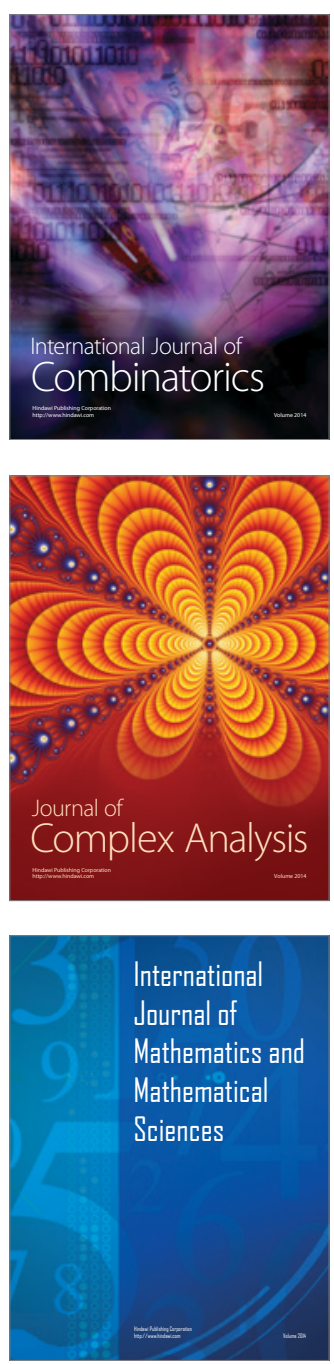
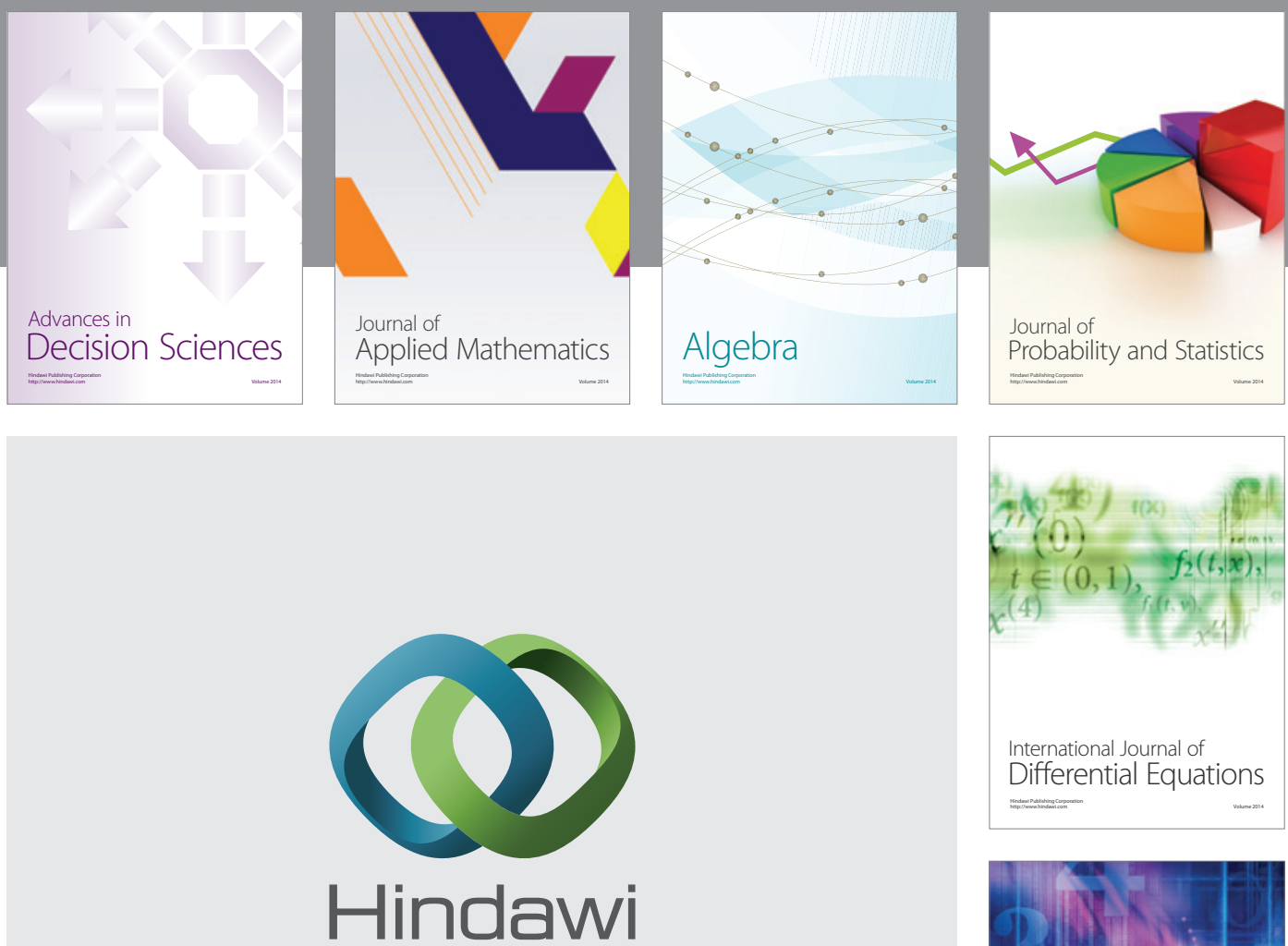

Submit your manuscripts at http://www.hindawi.com
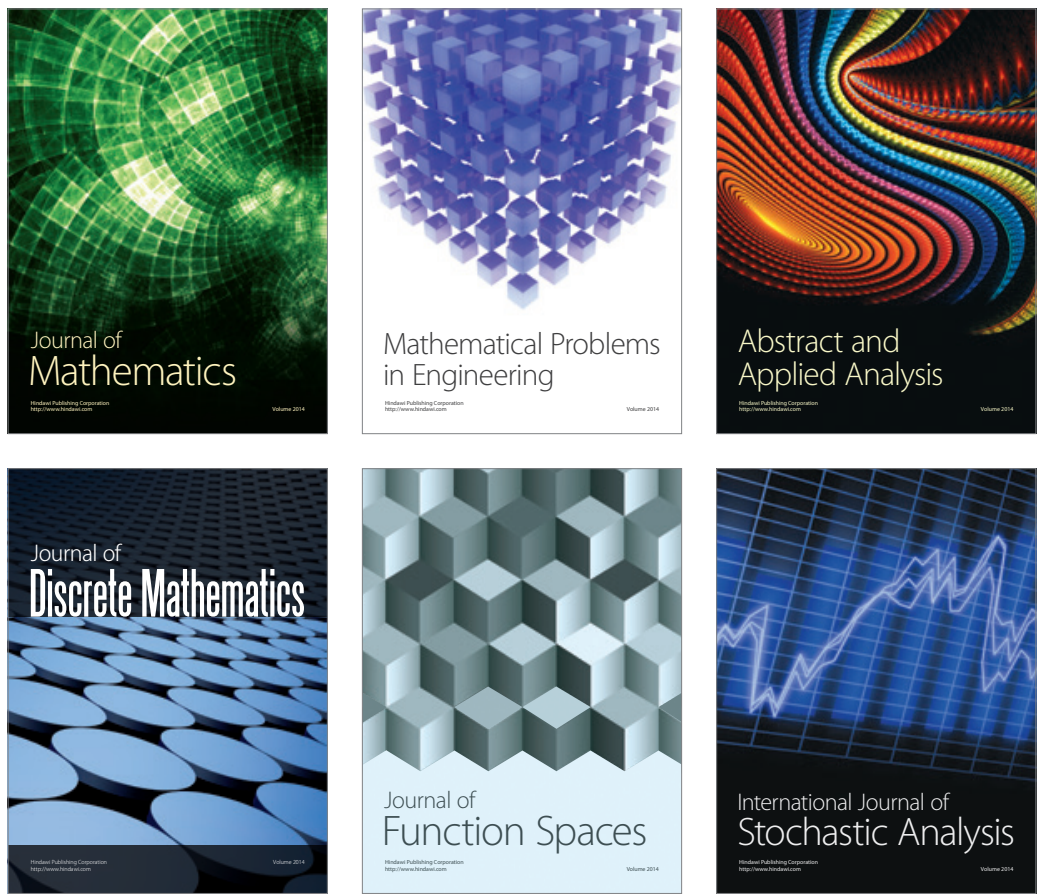

Journal of

Function Spaces

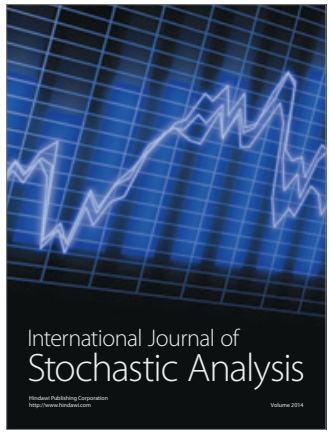

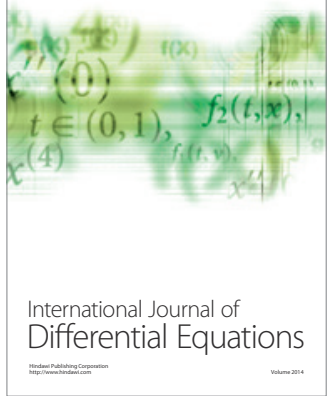
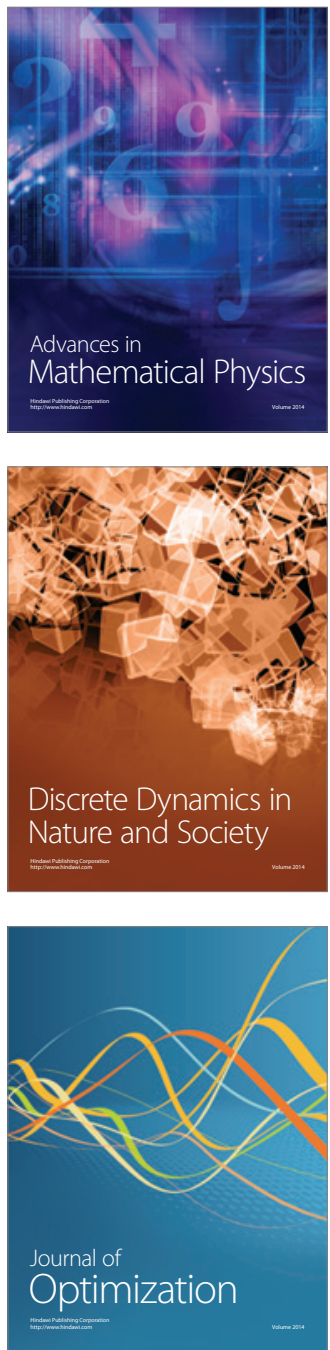